\title{
Long-Term Survival in a Patient With Ruptured Hepatocellular Carcinoma
}

\author{
Joon Sung Kim', Seung Kew Yoon', Jeong Ah Kim', Chang Wook Kim', \\ Si Hyun Bae', Jong Young Choi' and Chan-Kwon Jung ${ }^{2}$
}

Departments of 'Internal Medicine and 'Pathology, College of Medicine, The Catholic University of Korea, Seoul, Korea

\begin{abstract}
A 57-yr-old woman previously diagnosed with chronic hepatitis B was admitted via the emergency room because she suddenly developed epigastric pain with abdominal distension. On computed tomography (CT), a round enhancing mass was found on the left hepatic lobe with ascites in the peritoneal space. Bloody ascites were found upon tapping the ascites, and this led to the diagnosis of ruptured hepatocellular carcinoma (HCC). The patient was immediately treated with transcatheter arterial chemoembolization (TACE) including $50 \mathrm{mg}$ of adriamycin and $10 \mathrm{~mL}$ of lipiodol, and then we performed left lateral segmentectomy 20 days later. To prevent recurrence of $\mathrm{HCC}$ by any micrometastasis, the patient subsequently received 8 cycles of adjuvant systemic chemotherapy (a regimen of epirubicin $\left(50 \mathrm{mg} / \mathrm{m}^{2}\right)$, cisplatin $\left(60 \mathrm{mg} / \mathrm{m}^{2}\right)$ and 5 -fluorouracil $\left.\left(200 \mathrm{mg} / \mathrm{m}^{2}\right)\right)$ at monthly intervals. After this, the patient has been regularly followed up and she shows no signs of tumor recurrence 7 years later. This case suggests that surgical resection and subsequent adjuvant systemic chemotherapy with using an ECF regimen may provide long-term survival for patients ruptured HCC. (Korean $\mathbf{J}$ Intern Med 2009;24:63-67)
\end{abstract}

Keywords: Hepatocellular carcinoma; Spontaneous rupture; Hepatic resection; Chemotherapy; Prognosis

\section{INTRODUCTION}

Hepatocellular carcinoma (HCC) is one of the most common malignancies worldwide. In Korea, HCC is the third most common primary malignant tumor and the third leading cause of death in patients with cancer [1]. Spontaneous rupture of HCC is an uncommon, but fatal complication leading to immediate death, and especially for those patients with liver cirrhosis and who may have coagulation deficiencies. The incidence of spontaneous HCC rupture is different according to the geographical area, and it tends to be higher in areas with endemic viral hepatitis B or C, such as Korea [2-4].

In general, the diagnosis of ruptured HCC is based on the findings of ultrasound or CT, and on the presence of bloody ascites as ascertained by performing abdominal paracentesis. Regarding to the management for ruptured HCC, various treatment options, including hepatic resection, hepatic artery ligation and transcatheter arterial embolization (TAE), have been proposed in accordance with the situation/state the patient is in. When hypovolemic shock occurs due to massive intraperitoneal hemorrhage, immediate management such as resuscitation for hypovolemic shock followed by hemostasis is required before considering a proper treatment for the underlying HCC. Although hepatic resection is known to be the most effective treatment for ruptured HCC, the mortality rate for emergency operations in the ruptured patients with hepatic decompensation or hypovolemic shock has been high. TAE is alternatively considered to reduce the initial 

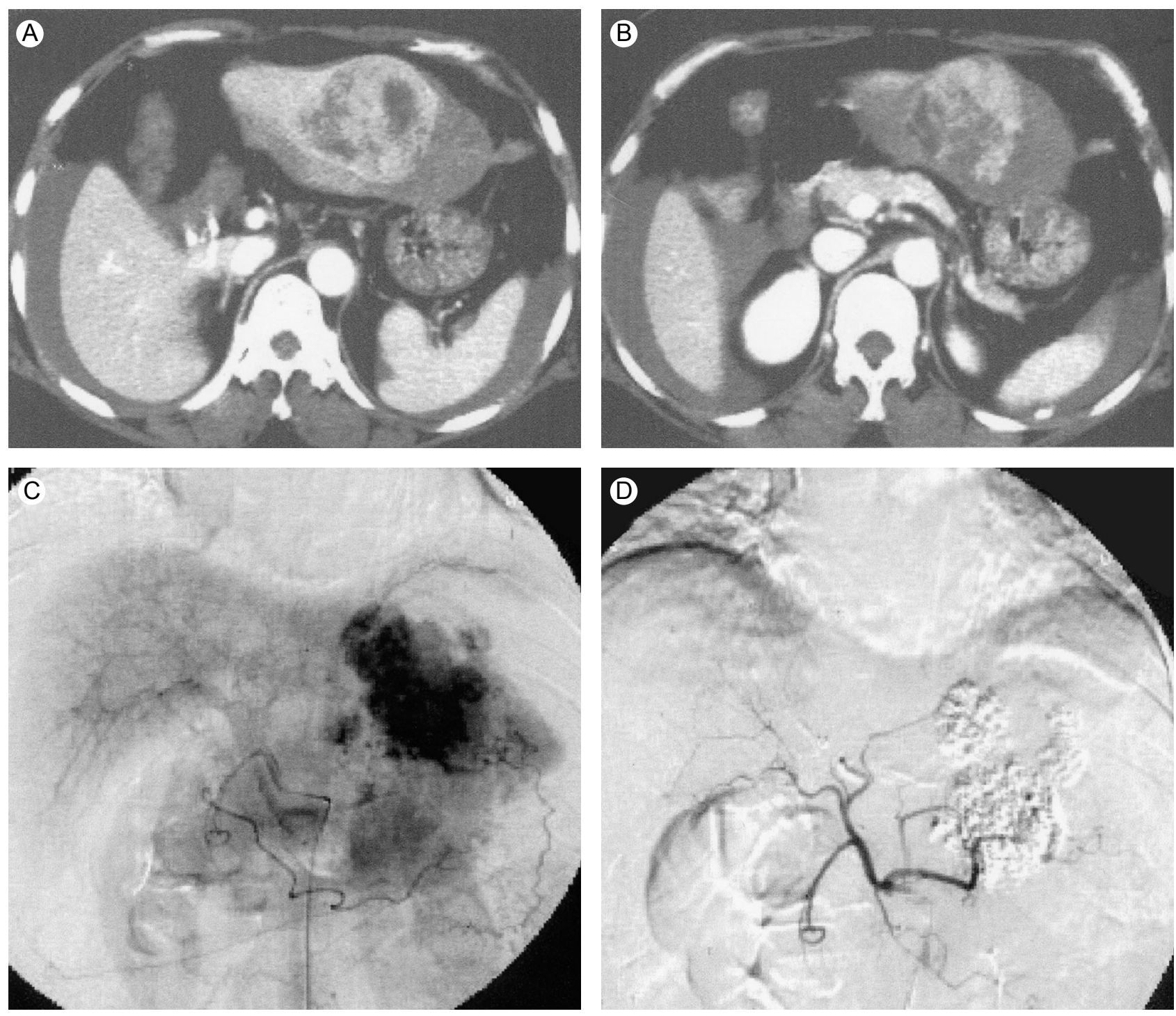

Figure 1. Abdominal enhanced CT scan shows a large arterial-phase enhancing mass in the left lobe of the liver, along with ascites (A\&B). Selective hepatic arteriography shows hypervascular tumor stains in the left lobe of the liver (C\&D).

mortality and to buy a little time for making the decision on the proper treatment for the underlying HCC. A previous study reported the average survival times for patients treated with emergency resection or with delayed resection were 12 months and 19 months, respectively [5]. However, HCC recurred in most of the patients treated by resection [5]. Therefore, an additional therapeutic modality is needed to treat any and all micro-metastatic tumor that could exist after ruptured HCC. Herein we report on a case of ruptured HCC that was treated with hepatic resection and subsequent chemotherapy, and the patient continues to enjoy her life 7 years after the operation.

\section{CASE REPORT}

A 57-yr-old woman was admitted to our hospital because of sudden-onset abdominal pain. She was a known chronic HBV carrier. She had no significant medical history such as diabetes, hypertension or tuberculosis; she did not smoke or drink any alcohol. On admission, she had a blood pressure of 90/60 $\mathrm{mmHg}$, a $105 / \mathrm{min}$ pulse rate, a $20 / \mathrm{min}$ respiratory rate and a body temperature of $36.3{ }^{\circ} \mathrm{C}$. On physical examination, the patient had an acutely ill looking appearance with abdominal distension and she felt tenderness on the whole abdomen upon palpation. The laboratory data on admission showed a white blood cell count of $5,800 / \mathrm{mm}^{3}$, a hemoglobin level of $10.8 \mathrm{~g} / \mathrm{dL}$, a hematocrit of $30.6 \%$ and a platelet count of $123,000 / \mathrm{mm}^{3}$. The serum alanine 

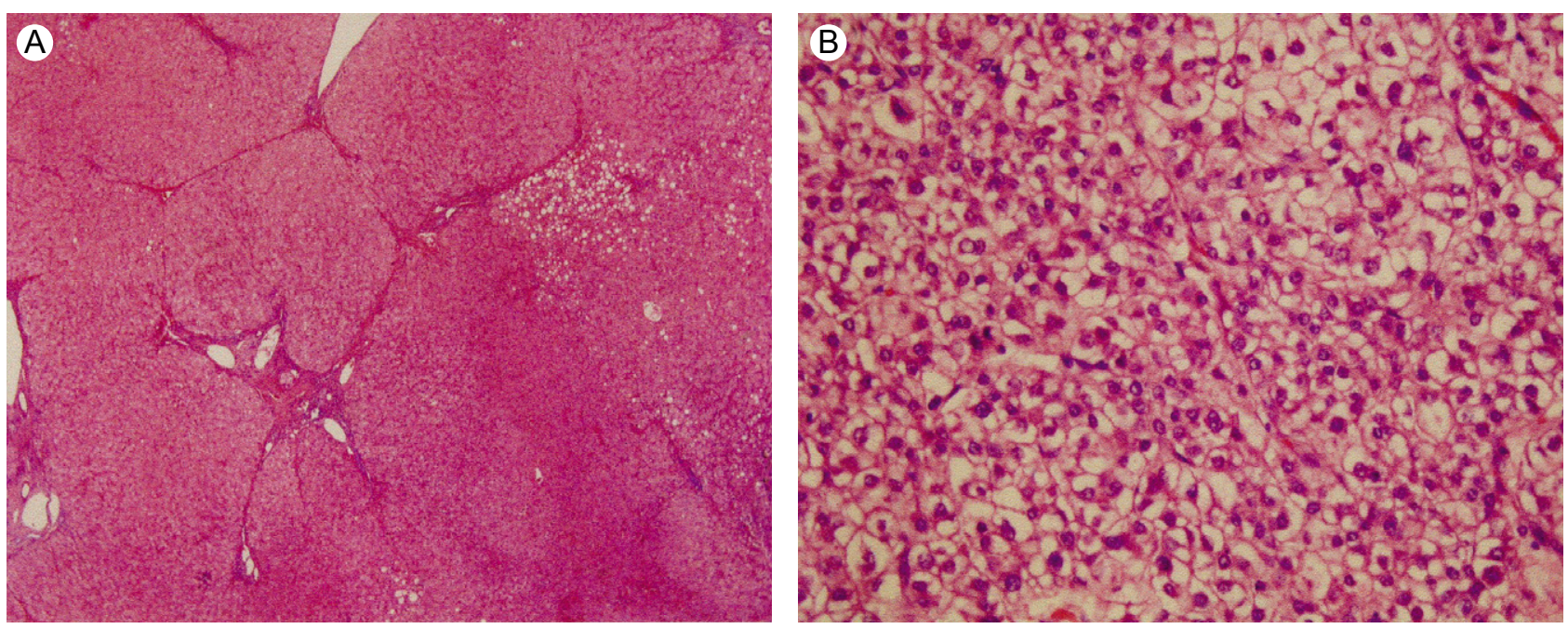

Figure 2. Liver Biopsy. (A) The liver shows mixed micro-macronodular cirrhosis with foci of micro-and macrovesicular steatosis (H\&E, original magnification $\times 40$ ). (B) The liver specimen shows a grade ll hepatocellular carcinoma, according to the criteria of Edmondson- Steiner. The tumor cells have abundant cytoplasm, and the cells are polygonal in shape. There are predominate nuclei and nucleoli (H\&E, original magnification $\times 400)$.

transaminase (ALT) was $72 \mathrm{U} / \mathrm{L}$ (normal: 5-40 U/l), the serum aspartate transaminase (AST) was $122 \mathrm{U} / \mathrm{L}$ (normal: 8-40 IU/L), the total protein $6.5 \mathrm{~g} / \mathrm{dL}$, the serum albumin $3.6 \mathrm{~g} / \mathrm{dL}$ and the alkaline phosphatase $187 \mathrm{U} / \mathrm{L}$ (normal: 100-250 U/L). The prothrombin time was 100\% (INR, 0.95) and the total bilirubin level was $4.8 \mathrm{mg} / \mathrm{dL}$ (direct bilirubin $1.7 \mathrm{mg} / \mathrm{dL}$ ). She was positive for $\mathrm{HBsAg}$, but negative for HBeAg, and the HBV DNA level was less than $2.5 \mathrm{pg} / \mathrm{mL}$. The level of alpha-fetoprotein (AFP) was $62.3 \mathrm{ng} / \mathrm{mL}$ (normal: $0-20 \mathrm{ng} / \mathrm{mL}$ ). The chest X-ray showed elevation of the right hemidiaphragm and abdominal X-ray showed abdominal distension, centralization and floating of bowel loops and widening of the interloopal space, which suggested ascites. Abdominal enhanced computerized tomography (CT) scanning showed a large arterial-phase enhancing mass in the left lobe with ascites (Fig. 1A and 1B).

She was immediately treated with transarterial chemoembolization (TACE) by a regimen of adriamycin $50 \mathrm{mg}$ and lipiodol $10 \mathrm{~mL}$ (Fig. $1 \mathrm{C}$ and $1 \mathrm{D}$ ). We performed left lateral segmentectomy twenty days after the TACE (Fig. 2A and 2B). One month after the hepatic surgery, to prevent recurrence of HCC by micro-metastasis, the patient subsequently received 8 cycles of adjuvant systemic chemotherapy by a regimen of epirubicin (50 $\mathrm{mg} / \mathrm{m}^{2}$ ), cisplatin $\left(60 \mathrm{mg} / \mathrm{m}^{2}\right)$ and 5 -fluorouracil (5-FU, $\left.200 \mathrm{mg} / \mathrm{m}^{2}\right)$ at monthly intervals. After this, patient has been regularly followed up to date and she shows no signs of tumor recurrence 7 years later (Fig. 3). This case suggests that surgical resection and subsequent adjuvant systemic chemotherapy using an ECF regimen may provide long-term survival for patients with ruptured HCC.

\section{DISCUSSION}

The ruptured HCC is a life threatening condition that may cause severe bleeding, hypotension and hypoperfusion of the liver, especially in cirrhotic patients who have coagulation deficiencies. Therefore, the mortality for this catastrophic event is very high, and early diagnosis of the ruptured HCC is essential to prolong the survival of these patients. The most characteristic finding of ruptured HCC is reported to be the sudden-onset of abdominal pain [6] and a physician, when observing such symptoms in geographic areas that are endemic for hepatitis viruses, can have a strong suspicion of ruptured HCC.

The mechanism of HCC rupture is poorly understood. However, several factors such as direct trauma, high intraluminal pressure due to rapid growth of tumor, necrosis, rupture by splitting of the normal hepatic parenchyma or erosion of a vessel, occlusion of the hepatic veins by tumor thrombus, and coagulopathy have been accepted as causes $[7,8]$. In general, tumors that protrude beyond the original liver contour may rupture more readily than those that are surrounded by normal parenychma [9]. Furthermore, a peripheral location and protrusion of the tumor or discontinuity of the hepatic surface, and poor liver function should be recognized as high risk factors for HCC rupture. However, the TMN stage, the presence of portal vein thrombosis and the size of the tumor seem to 

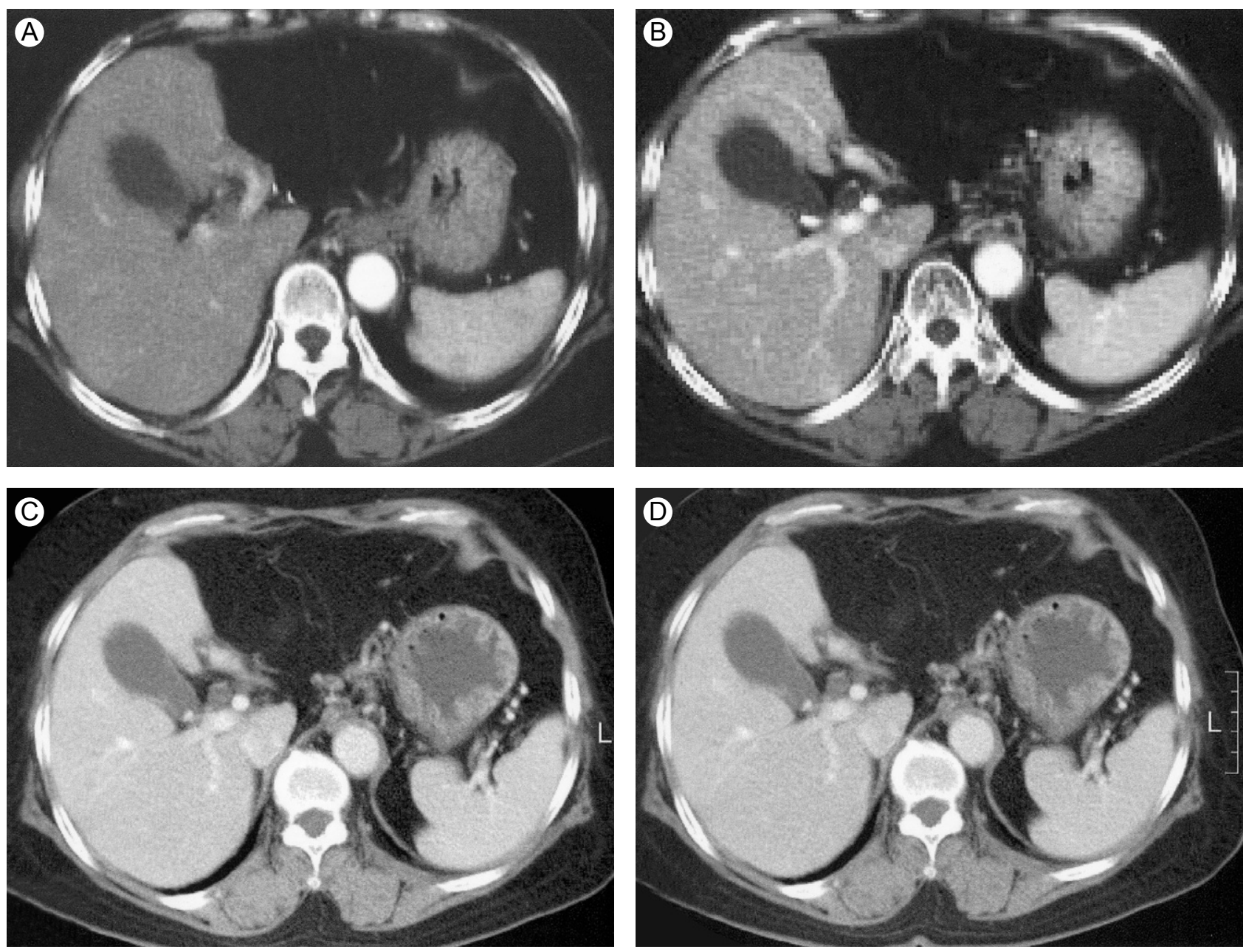

Figure 3. CT findings during the follow up period after surgical resection of the left lobe and the subsequent chemotherapy. There is no evidence of tumor recurrence on the arterial (A) and portal phase (B) of the dynamic CT scan that was done 1 month after the operation. Seven and a half years after the initial treatment, the arterial (C) and portal phase (D) images show no abnormal tumor staining.

be irrelevant to HCC rupture [10]. Rupture of $\mathrm{HCC}$ is confirmed by performing abdominal paracentesis, and the fluid shows blood-stained ascites. However, an imaging study such as ultrasonography or CT can demonstrate the extent of blood loss, the severity of the liver cirrhosis, the location of the tumor and the patency of the portal vein [11].

The primary purpose of treatment for ruptured HCC is to stabilize the hypovolemic shock that's due to massive hemorrhage. Next, the treatment of the underlying HCC using various modalities, including hepatic resection or TAE, should be considered. Yet the feasibility of performing hepatic resection is dependent on the hepatic reserve and the underlying liver cirrhosis. Previous studies have demonstrated that the resectability rates of ruptured HCC patients ranged from $12.5 \%$ to $59.3 \%[6,12]$. In addition, the survival rate was greater in the delayed resection group compared to the emergency resection group (19 months vs. 12 months, respectively) [5]. However, the longest survival in the emergency group was 33 months and this was 27 months in the delayed resection group [5]. In this regard, delayed resection seems to improve the short-term survival, but the long-term survival remains questionable. In the present case, the patient immediately received TACE after the diagnosis of ruptured HCC, and this was followed by left lateral segmentectomy. Although the efficacy of chemotherapy for HCC is still debatable, our previous study showed a significant anti-tumor effect in selected patients with HCC [13]. Based on this result and with the hope of preventing HCC recurrence, we treated the lady with 8 cycles of adjuvant systemic chemotherapy by a regimen of epirubicin, cisplatin and 5-fluorouracil at monthly intervals. After this, the patient has been regularly followed up to date and she shows no signs of tumor recurrence 7 years later. Few cases have been reported in the medical literature for which the patients lived 7 years 
or more after experiencing ruptured HCC, such as our patient has done. The reason why our patient has survived for such a long time without recurrence of HCC may be attributed to the adjuvant chemotherapy we aggressively administered after surgical resection. In addition, her good liver function and lower viral load may have contributed to the good prognosis. It is essential that the chosen treatment be tailored to a ruptured HCC patient's individual needs to prolong the survival time. In summary, performing surgical resection and subsequently adjuvant systemic combination chemotherapy may provide a fighting chance for patients with ruptured HCC to achieve long-term survival.

\section{REFERENCES}

1. Korea National Statistical Office (KNSO). Avail from: http:// www.nso.go.kr

2. Ong GB, Taw JL. Spontaneous rupture of hepatocellular carcinoma. Br Med J 1972;4:146-149.

3. Chearanai O, Plengvanit U, Asavanich C, Damrongsak D, Sindhvananda K, Boonyapisit S. Spontaneous rupture of primary hepatoma: report of 63 cases with particular reference to the pathogenesis and rationale treatment by hepatic artery ligation. Cancer 1983;51:1532-1536.

4. Clarkston W, Inciardi M, Kirkpatrick S, McEwen G, Ediger S, Schubert T. Acute hemoperitoneum from rupture of a hepatocellular carcinoma. J Clin Gastroenterol 1988;10:221-225.

5. Buczkowski AK, Kim PT, Ho SG, et al. Multidisciplinary management of ruptured hepatocellular carcinoma. J
Gastrointest Surg 2006;10:379-386.

6. Yeh CN, Lee WC, Jeng LB, Chen MF, Yu MC. Spontaneous tumour rupture and prognosis in patients with hepatocellular carcinoma. Br J Surg 2002;89:1125-1129.

7. Liu CL, Fan ST, Lo CM, et al. Management of spontaneous rupture of hepatocellular carcinoma: single-center experience. J Clin Oncol 2001;19:3725-3732.

8. Terada T, Takeuchi T, Hirano R, Nagata S, Kubota H, Honda S. Spontaneous rupture of peripancreatic lymph node with hepatocellular carcinoma metastasis: report of an autopsy case with massive peritoneal bleeding. Hepatol Res 2003;26:73-76.

9. Choi BG, Park SH, Byun JY, Jung SE, Choi KH, Han JY. The findings of ruptured hepatocellular carcinoma on helical CT. Br J Radiol 2001;74:142-146.

10. Min HJ, Lee OJ, Kang DY, et al. The clinical study on spontaneously ruptured hepatocellular carcinoma. Korean $\mathrm{J}$ Gastroenterol 2004;44:160-167.

11. Chou YH, Chiou HJ, Tiu CM, Chen JD, Hung GS, Chiang JH. Some unusual complications of malignancies: case 1. spontaneous rupture of hepatocellular carcinoma demonstrated by contrastenhanced sonography. J Clin Oncol 2002;20:4108-4111.

12. Lai EC, Wu KM, Choi TK, Fan ST, Wong J. Spontaneous ruptured hepatocellular carcinoma: an appraisal of surgical treatment. Ann Surg 1989;210:24-28.

13. Jang JW, Park YM, Bae SH, et al. Therapeutic efficacy of multimodal combination therapy using transcatheter arterial infusion of epirubicin and cisplatin, systemic infusion of 5fluorouracil, and additional percutaneous ethanol injection for unresectable hepatocellular carcinoma. Cancer Chemother Pharmacol 2004;54:415-420. 